EPJ Web of Conferences 41, 02003 (2013)

DOI: $10.1051 /$ epjconf/20134102003

C Owned by the authors, published by EDP Sciences, 2013

\title{
Attosecond streaking of shake-up and Auger electrons in xenon
}

\author{
A.J. Verhoef ${ }^{1}$, A. Mitrofanov ${ }^{1}$, M. Krikunova ${ }^{2,3}$, N.M. Kabachnik ${ }^{4,5}$, M. Drescher ${ }^{2}$, and A. Baltuska ${ }^{1}$ \\ ${ }^{1}$ Photonics Institute, Technische Universität Wien, Gusshausstrasse 27-29/387, 1040 Vienna, Austria \\ ${ }^{2}$ Institut für Experimentalphysik, Universität Hamburg, Luruper Chaussee 149, 22761 Hamburg, \\ Germany \\ ${ }^{3}$ Institut für Optik und Atomare Physik, Technische Universität Berlin, Hardenbergstrasse 36, 10623 \\ Berlin, Germany \\ ${ }^{4}$ Institute of Nuclear Physics, Moscow State University, Moscow 119991, Russia \\ ${ }^{5}$ European XFEL GmbH, Albert-Einstein-Ring 19, 22761 Hamburg, Germany
}

\begin{abstract}
We present first results of simultaneous attosecond streaking measurements of shake-up electrons and Auger electrons emitted from xenon. We extract relative photo-emission delays for electrons emitted from the $4 \mathrm{~d}, 5 \mathrm{~s}$ and $5 \mathrm{p}$ subshell, as well as for the $5 \mathrm{p}^{-2} 5 \mathrm{~d}$ correlation satellite (shake-up electrons).
\end{abstract}

The recent advances in attosecond physics revealing an emission delay between electrons emitted from valence states and deeper bound $4 \mathrm{f}$ states in tungsten [1] and from the $2 \mathrm{p}$ and $2 \mathrm{~s}$ subshells in neon [2] have provoked and will continue to provoke questions about the physics of photoionization. However, in the aforementioned experiments a relative phase ambiguity remained, because of the spectral separation of the respective emitted wavepackets. We present experimental data obtained using this method from an atomic system, xenon, from which more different electron wavepackets including shake-up electron wavepackets are emitted, and where a spectral overlap of some of these wavepackets can be observed. The spectral overlap of the different emitted electronic wavepackets can eliminate the relative phase ambiguity, and thus allows for a more reliable measurement of the relative emission phase and delay. Recently, attosecond spectroscopic measurements from xenon using a velocity map imaging (VMI) spectrometer were reported [3], revealing a weak phase sensitivity of sideband generation of the Auger emission, in agreement with numerical simulations presented previously [4]. Although VMI has the advantage that the angular distribution of the electrons can be obtained, the spectral resolution was much lower than that of our linear time-of-flight spectrometer [5], such that the individual Auger lines could not be resolved and no information about the valence electrons was obtained. In the experimental data we present here, the Auger lines and corresponding sidebands are individually resolved, and the streaking of the 5p, $5 \mathrm{~s}$ and $4 \mathrm{~d}$ photoelectrons and the $5 \mathrm{p}^{-2} 5 \mathrm{~d}$ shake-up satellite is well resolved.

Carrier envelope phase (CEP) stable 5-fs pulses with a central wavelength of $\sim 750 \mathrm{~nm}$ and $\sim 250$ $\mu \mathrm{J}$ energy are generated by focusing $700 \mu \mathrm{J}, 25$ fs pulses from a CEP stabilized Ti:sapphire multipass amplifier in a neon-filled capillary and subsequent compression in a chirped mirror compressor. Through loose focusing of the CEP stable few-cycle pulses on a neon target, highharmonics are generated. The few-cycle pulses are spatially separated from the generated harmonics using an annular filter consisting of a pellicle with a central hole that is covered with a $\mathrm{Zr}$ foil.

This is an Open Access article distributed under the terms of the Creative Commons Attribution License 2.0, which permits unrestricted use, distribution, and reproduction in any medium, provided the original work is properly cited. 

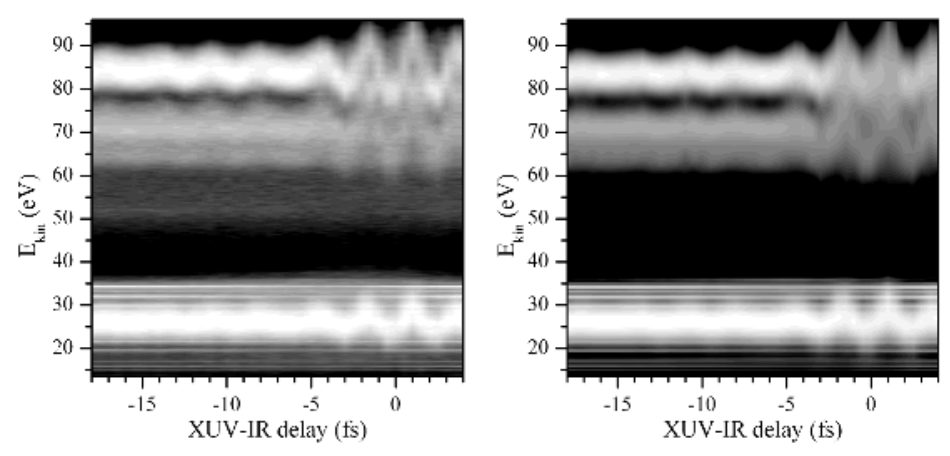

Fig. 1. Measured (left) and fitted (right) spectrogram from xenon. Negative delay means the attosecond pump pulse arrives before the few-cycle probe pulse. The weak spectral features between 50 and $60 \mathrm{eV}$ are not considered in the fit, because the signal to noise ratio of these features around zero delay is too small. In the experimental data, a second order sideband to the Auger lines, which we have not considered in the fit, can be observed as well.
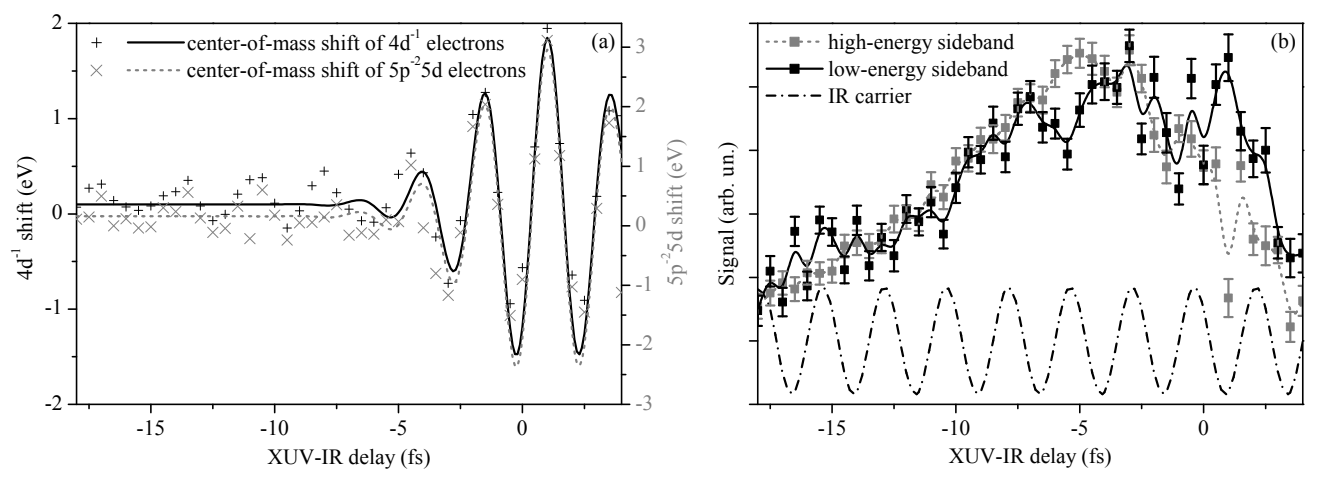

Fig. 2. (a) Center of mass analysis of the $4 d$ (black) and $5 p^{-2} 5 d$ (gray) features. No delay between the two features can be observed. The $5 \mathrm{p}$ and $5 \mathrm{~s}$ features are omitted for clarity. (b) Strength of the up- (gray) and downshifted (black) sidebands to the Auger lines. A weak 180 degrees out of phase oscillation of the sidebands can be observed. For clarity, the IR carrier oscillation is indicated by the black dash-dotted line

Through spectral filtering using the $150 \mathrm{~nm}$ thin $\mathrm{Zr}$ foil and a spherical Mo/Si multilayer double mirror the continuum in the cut-off region is selected, resulting in an isolated attosecond pulse with a central photon energy of $94 \mathrm{eV}$ and a full width at half maximum of $6 \mathrm{eV}$. The delay between the attosecond pulse and the few-cycle pulse is controlled via translation of the inner component of the double mirror. The double mirror focuses both pulses on a xenon gas jet, and the ejected photoelectrons are analyzed using a time-of-flight (TOF) spectrometer.

In Figure 1 we show the measured and fitted attosecond streaking spectrograms. In order to extract information about the evolution of the different emitted electrons, we fitted the spectrum for each delay, as in [5], obtaining the following parameters: The intensity of the main Auger lines, the intensity of the corresponding up-shifted sidebands and of the corresponding down-shifted sidebands, as well as the width and center-of-mass of the direct photo-electron features. The results of this analysis are presented in Figure 2. Similarly to Ref [3], we observe a weak phase-sensitive behaviour of the sidebands to the Auger emission. Comparing the changes in center of mass of the photoelectron features as a function of pump probe delay can yield information on possible photoemission delay [1]. Following this strategy, our experimental data shows no delay between the photoemission from the $5 \mathrm{p}(83 \mathrm{eV}), 5 \mathrm{~s}(72 \mathrm{eV})$ and $4 \mathrm{~d}(27 \mathrm{eV})$ electrons or the $5 \mathrm{p}^{-2} 5 \mathrm{~d}$ shake-up satellite $(65 \mathrm{eV})$ (see Figures 1 and 2). Because of our rather coarse step size, and accuracy of \pm 15 as for the pump-probe delay, we infer an upper limit of 50 as for the photoemission delay in the aforementioned spectral features. In a bid to obtain a better resolution for the extracted photo- 


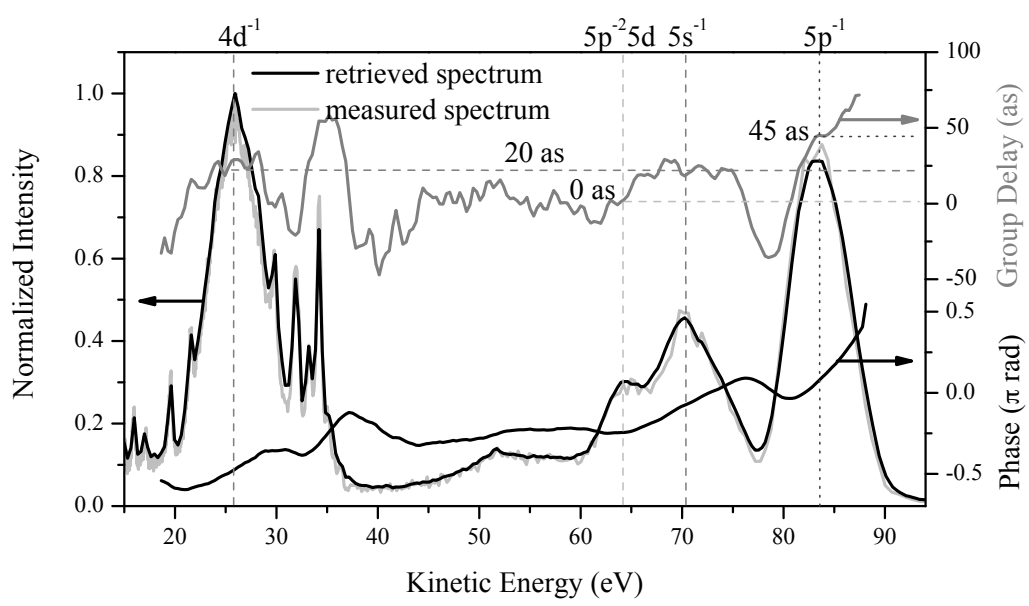

Fig. 3. Spectral phase and group delay obtained from the FROG-CRAB analysis of the spectrogram over the delay range from -18 to $-6 \mathrm{fs}$, revealing no delay $(0 \pm 10$ as $)$ between the $4 \mathrm{~d}$ and $5 \mathrm{~s}$ electrons. A separate, more accurate analysis of only the higher-energy part of the spectrum $(>55 \mathrm{eV})$ yielded a delay of -20 as for the $5 \mathrm{p}^{-}$

${ }^{2} 5 \mathrm{~d}$ electrons, 0 as for the $5 \mathrm{~s}$ electrons and +30 as for the $5 \mathrm{p}$ electrons.

emission delays, FROG-CRAB analysis was carried out. For technical reasons the spectrogram around zero delay had to be excluded from the FROG-CRAB analysis. The result of the FROGCRAB analysis is shown in Fig. 3, and a comparison with the known measurement induced energy dependent delay is presented in Table 1.

We presented high-resolution attosecond streaking measurements in xenon that can give more insight in possible delays in atomic photo-ionization. Our data reveals a weak phase sensitivity of the sideband formation to the Auger emission lines, similarly to recent experimental observations [3], and in agreement with previous numerical simulations [4]. FROG-CRAB analysis of the measured spectrogram reveals that the $5 \mathrm{p}^{-2} 5 \mathrm{~d}$ shake-up emission precedes the emission from the $4 \mathrm{~d}$ and $5 \mathrm{~s}$ subshells by $\sim 20$ as, and that the emission from the $5 \mathrm{p}$ subshell is delayed by $\sim 30$ as with respect to the emission from the $4 \mathrm{~d}$ and $5 \mathrm{~s}$ subshells.

Table 1. Measured delays relative to the $5 \mathrm{~s}$ emission compared to the measurement induced (Coulomb-laser coupling - CLC) delay relative to the $5 \mathrm{~s}$ emission. Negative delay means the emission appears to occur at earlier times.

\begin{tabular}{c|c|c|c|c}
$\begin{array}{c}\text { Spectral } \\
\text { feature }\end{array}$ & $\begin{array}{c}\boldsymbol{E}_{\text {kin }} \\
(\mathbf{e V})\end{array}$ & $\begin{array}{c}\text { Delay } \\
\text { meas. (as) }\end{array}$ & $\begin{array}{c}\text { Delay } \\
\text { CLC (as) }\end{array}$ & $\begin{array}{c}\text { Delay } \\
\text { diff. (as) }\end{array}$ \\
\hline $4 \mathrm{~d}^{-1}$ & 26 & $0 \pm 10$ & -18 & 18 \\
$5 \mathrm{p}^{-2} 5 \mathrm{~d}$ & 64 & $-20 \pm 5$ & -1 & -19 \\
$5 \mathrm{~s}^{-1}$ & 70 & 0 & 0 & 0 \\
$5 \mathrm{p}^{-1}$ & 83 & $30 \pm 5$ & 2 & 28
\end{tabular}

\section{References}

1. A. Cavalieri et al., Nature 449, 1029 (2007)

2. M. Schutze et al., Science 328, 1658 (2010)

3. S. Zherebtsov et al., J. Phys. B 44, 105601 (2011)

4. M. Drescher et al., Nature 419, 803 (2002)

5. A.J. Verhoef et al., New J. Phys 13, 113003 (2011) 